\title{
Role of resistivity gradient in laser-driven ion acceleration
}

\author{
L. A. Gizzi, ${ }^{1,2, *}$ S. Betti, ${ }^{1,2, \dagger}$ E. Förster, ${ }^{3, \ddagger}$ D. Giulietti, ${ }^{1,2, \S}$ S. Höfer, ${ }^{3, \sharp}$ P. Köster, ${ }^{1,2}$ L. Labate, ${ }^{1,2}$ \\ R. Lötzsch, ${ }^{3,+}$ A. P. L. Robinson, ${ }^{4}$ and I. Uschmann ${ }^{3}$ \\ ${ }^{1}$ ILIL, Istituto Nazionale di Ottica, UOS “Adriano Gozzini,” CNR, Via G. Moruzzi 1, Pisa, Italy \\ ${ }^{2}$ INFN, Sezione di Pisa, Largo B. Pontecorvo 3, Pisa, Italy \\ ${ }^{3}$ Institut für Optik und Quantenelektronik, Friedrich-Schiller-Universität, Max-Wien-Platz, 1, 07743 Jena, Germany \\ ${ }^{4}$ Central Laser Facility, Rutherford Appleton Laboratory, Chilton, OX11 OQX, United Kingdom
}

(Received 21 January 2010; published 19 January 2011)

\begin{abstract}
It was predicted that, when a fast electron beam with some angular spread is normally incident on a resistivity gradient, magnetic field generation can occur that can inhibit beam propagation [A. R. Bell et al., Phys. Rev. E 58, 2471 (1998)]. This effect can have consequences on the laser-driven ion acceleration. In the experiment reported here, we compare ion emission from laser irradiated coated and uncoated metal foils and we show that the ion beam from the coated target has a much smaller angular spread. Detailed hybrid numerical simulations confirm that the inhibition of fast electron transport through the resistivity gradient may explain the observed effect.
\end{abstract}

DOI: 10.1103/PhysRevSTAB.14.011301

PACS numbers: 52.38.Kd, 41.75.Jv, 52.25.Fi

Generation and transport of so-called fast electrons driven by ultraintense laser pulses are the key to inertial confinement fusion (ICF) in the fast ignition approach [1]. Fast electrons also provide the basic mechanism for emission of $K_{\alpha}$ ultrashort x-ray pulses [2-4]. Moreover, fast electrons play a crucial role [5-9] in laser-driven ion acceleration with the target normal sheath acceleration (TNSA) mechanism that has gained considerable attention because of the extremely diversified potential applications among which ICF with proton driven fast ignition [10], formation of high energy density states of matter [11] and proton imaging [12] are the most prominent ones. In all these experiments fast electron currents are well above the Alfven limit [13], and therefore transport strongly depends upon the conductivity of the medium [14] which must support propagation by supplying a balancing cold electron return current. In dielectrics these conditions are not easily fulfilled and inhibition of the fast electron transport may occur [15] leading to filamentation. A similar situation occurs in a low density material, like a gas target [16] or a low density foam [17], due to the lack of background electrons required to establish the return current.

Dedicated studies on ion acceleration from dielectric targets $[10,18]$ show that $\mu$ m-thick plastic coatings may

\footnotetext{
*la.gizzi@ino.it

${ }^{\dagger}$ Also at Accademia Navale, Livorno, Italy, and at Dipartimento di Ingegneria delle Telecomunicazioni, Università di Pisa, Italy.

${ }^{\ddagger}$ Also at Helmholtz Institute Jena, Helmholtzweg 4, 07743 Jena, Germany.

${ }^{\S}$ Also at Dipartimento di Fisica, Università di Pisa, Pisa, Italy.

Published by American Physical Society under the terms of the Creative Commons Attribution 3.0 License. Further distribution of this work must maintain attribution to the author(s) and the published article's title, journal citation, and DOI.
}

be sufficient to disrupt the uniformity of the ion beam due to filamentation of the fast electron beam in the plastic layer. Similar effects also occur in metals as shown in [19], where a strong collimating self-generated resistive magnetic field is invoked to explain the small half-angle divergence measured experimentally. These effects are expected to be enhanced in the case of coated or layered targets where jumps of resistivity exist along the main beam propagation direction. In a theoretical study [20] of laser interaction with layered targets, it was shown that the propagation of fast electrons across layers with different resistivity gives rise to the growth of a magnetic field at the interface. For strongly two-dimensional irradiation geometries, fast electrons propagating across the interface exhibit a component of the vector flux $\mathbf{j}_{\mathbf{f}}$ that is parallel to the target interface and therefore perpendicular to the resistivity gradient $\nabla \eta$. These circumstances lead to the generation of a magnetic field originating from the term $\mathbf{j}_{\mathbf{f}} \times \nabla \eta$. Because of the symmetry of the system, the magnetic field vanishes on the axis of the electron beam and fast electrons can leak through the axis. This is a signature that makes the identification of this mechanism easier in experiments. This scenario is different from the fast electron guiding targets proposed in [21] and recently investigated in [22] in which the resistivity gradients are constructed so as to be perpendicular to the passage of the beam. The case considered here is equally important for the understanding of fast electron transport and possible control of energy deposition in the fast ignition scenario [1].

In this context, reduction of the proton acceleration was claimed [23] when a thin plastic coating was used at the rear side of a $50 \mu \mathrm{m}$ thick aluminum target. In that experiment, a systematic study was carried out and the observed reduction of ion production was attributed to the metaldielectric interface due to the growth of a magnetic field in 
combination with expansion of the plastic layer due to surface heating. In our case we show hereby that the main difference in proton emission between coated and uncoated targets is not in the reduction of the proton yield, but in the transverse size of the proton emission. Both effects are predicted by theory and have important consequences on the interpretation in terms of the model of Ref. [20].

In a precursor experiment [24], a highly adhesive dielectric layer consisting of lacquer coating was used, to our knowledge for the first time, to study fast electron transport and proton acceleration. In that experiment, metal foils coated with lacquer were irradiated with ultrashort pulses at an intensity exceeding $10^{19} \mathrm{~W} / \mathrm{cm}^{2}$ and showed no evidence of filamentation of the fast electron current. In the same experiment, evidence of beam collimation was found, although direct comparison of coated and uncoated targets was not available.

In this paper we show the results of a new experiment in which a collimating effect induced by a thin dielectric layer was clearly visible for the first time for this class of experiments. In the experiment, $5.7 \mu \mathrm{m}$-thick, flat Ti foils, either uncoated or back-coated with the same lacquer adopted in [24], were irradiated with ultraintense pulses from the Jena 10 TW Ti:sapphire laser system (JETI). An $f / 1.2$ off-axis parabola was employed to focus 80 fs laser pulses down to a $5 \mu \mathrm{m}^{2}$ focal spot. The angle of incidence on the target was $10^{\circ}$. The average intensity on target was up to $5 \times 10^{19} \mathrm{~W} / \mathrm{cm}^{2}$, with a normalized vector potential $a_{0}=e A_{\mathrm{L}} / m_{e} c^{2} \approx 0.85 \lambda_{0} \sqrt{I_{\mathrm{L}}}=4.8, e$ and $m_{e}$ being the electron charge and mass, respectively, $A_{\mathrm{L}}$ the laser vector potential, $c$ the speed of light, $\lambda_{0}$ the laser wavelength in $\mu \mathrm{m}$, and $I_{\mathrm{L}}$ the intensity in units of $10^{18} \mathrm{~W} / \mathrm{cm}^{2}$.

Special attention was dedicated in our experiment to the preparation and characterization of the coated target. In fact, in this class of experiments, the quality of the interface between metal and dielectric may play an important role as discussed in [25]. Ordinary target plastic coating is usually based upon vacuum deposition. However, due to the weak adhesion of the plastic layer to the metal foil, mechanical stress due to a different thermal expansion coefficient may be sufficient to initiate layer separation. Also, the presence of a small precursor radiation on target can further increase layer separation [23]. In these circumstances, inhibition of the electron transport might occur due to vacuum gaps which may be present between different layers.

In our case, the coating consisted of a nitrocellulose lacquer, a material characterized by a high content of $\mathrm{H}$ and $\mathrm{C}$. The thickness of the coating was measured to be $1.5 \mu \mathrm{m}$ and its resistivity was found to be greater than $1.5 \times 10^{7} \Omega / \mathrm{m}$. Because of the established properties of lacquers, the coating should be regarded as a dielectric layer characterized by hardness, flexibility, and high adhesion to the substrate which ensures that no gap exists between the metal surface and the coating layer.
Proton detection was carried out using radiochromic films (RCF) which were placed behind the target at a distance of $3 \mathrm{~mm}$ and were shielded from direct laser radiation by a $20 \mu \mathrm{m}$ thick $\mathrm{Al}$ foil. The response of the RCF was fully characterized [26] using Monte Carlo simulations based upon the numerical code GEANT4 [27].

Figure 1 shows the typical patterns of the proton beam obtained in the case of uncoated (left) and coated (right) target. The image of Fig. 1 (left) shows an irregularly shaped cross section, with filament-like structures and an overall size of approximately $6 \mathrm{~mm}$ in diameter. Conversely, the image of Fig. 1 (right) shows a circular pattern, with no evidence of the filament-like structures found in the uncoated targets, and with a size of less than $3 \mathrm{~mm}$ that is, half of the size of the uncoated target case. The integrated optical density of Fig. 1 (left) is approximately twice that of Fig. 1 (right), while the peak intensity ratio between the optical densities of the two images is approximately 1.5 .

Both images of Fig. 1 were obtained from the first layer of the film stack which is sensitive to protons in the range from $U_{\min } \approx 1.2 \mathrm{MeV}$ to $U_{\max } \approx 3.5 \mathrm{MeV}$. $U_{\text {min }}$ was calculated using the code SRIM [28] by taking into account transmission through the $20 \mu \mathrm{m}$ thick Al foil placed in front of the RCF stack. $U_{\max }$ was obtained separately by performing a radiographic image of a Ta mesh made of a $35 \mu \mathrm{m}$-diameter wire as described in [24].
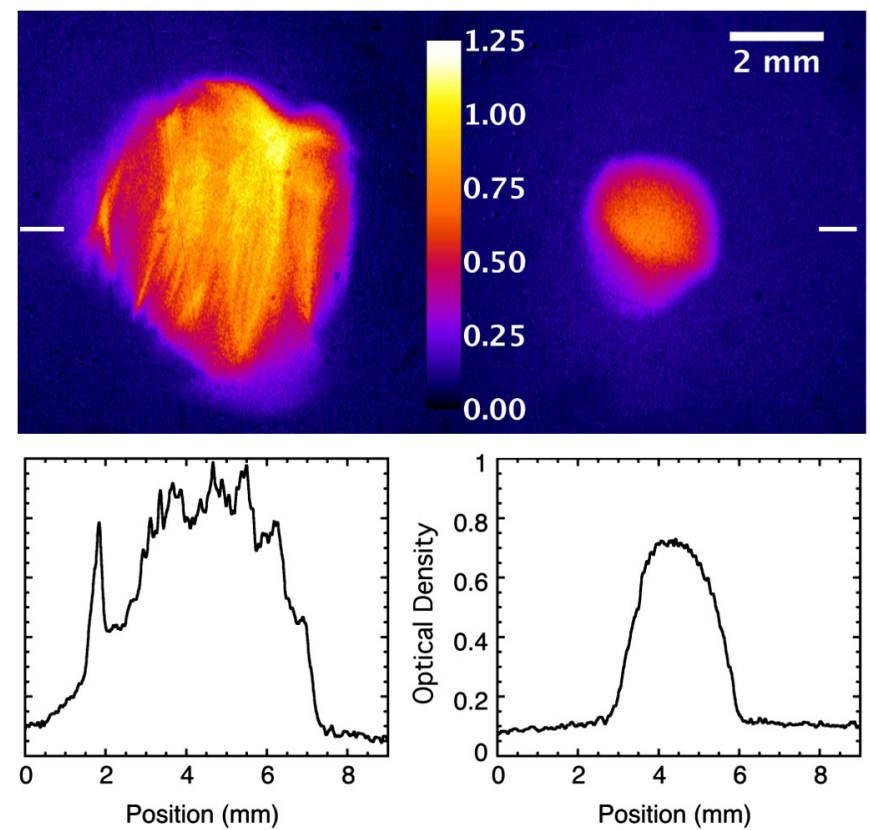

FIG. 1. Proton beam image after irradiation of uncoated (left) and dielectric rear-coated (right) $5.7 \mu \mathrm{m}$ thick Ti target under the same irradiation conditions. The image shows the optical density of the two RCF and the displayed color bar applies to both images. Also shown at the bottom of the figure are the lineouts of the two images taken along the diameters as indicated by the two dashes. 
These results clearly show that in our experimental conditions the thin rear surface dielectric coating has a major influence on the observed proton acceleration. This is a strong indication of the fact that, in our configuration, proton acceleration occurs at the target rear side as expected from the TNSA mechanism, ruling out contribution from the front side. The presence of the dielectric coating appears to collimate the proton beam and to suppress its intensity modulations. In principle, the observed collimation could be due either to a smaller aperture of proton emission cone or to a smaller transverse size of the emitting region. In our experiment, due to the short distance between the target and the RCF, this collimation was correlated strongly to the latter effect. According to the TNSA, this implies a more localized sheath field which is due to a narrower fast electron beam at the rear surface.

A possible general explanation for the collimation effect like the one presented here is the dependence of the proton beam divergence upon the proton energy. This effect has been predicted by TNSA models and numerical simulations [29] and has been confirmed by recent experiments [30]. However, these studies also confirm that, within the spectral range from 1.2 to $3.5 \mathrm{MeV}$ explored in our measurements, the proton emission angle is constant. Therefore, we cannot invoke changes in the spectral distribution of ions to explain the observed collimation.

These experimental observations are instead consistent with the following picture. In the case of coated targets, only the higher energy component of the fast electron bunch close to the propagation axis reaches the target rear side giving rise to a localized sheath field and accounting for the small spatial cross sections of the accelerated proton beam. In contrast, in the case of uncoated metal targets, the entire fast electron bunch, including the marginal (away from the axis) lower energy electrons, emerge from the target rear side contributing as a whole to the proton acceleration process. In the latter case, the nonuniformity of the proton image is a consequence of the resistive filamentation of the fast electrons inside the metal as discussed in [19].

We observe that some contribution to the nonuniformity may also come from the surface roughness of the uncoated metal foil which may affect the uniformity of the sheath field. The surface roughness of the uncoated foil was measured to be approximately $65 \mathrm{~nm}( \pm 10 \%)$ which is roughly 2 orders of magnitude below the size of the focal spot. These circumstances make us confident that the resistive filamentation should account for most of the observed proton beam nonuniformity, although, at this stage, we cannot exclude contribution from target surface roughness.

The scenario depicted above is qualitatively consistent with the behavior predicted in [20] for layered targets. For a semiquantitative description of this process we carried out numerical simulations of transport of the fast electrons in both uncoated and dielectric-coated metal targets using the 2D hybrid Vlasov-Fokker-Planck (VFP) numerical code LEDA [21]. The code uses a KALOS-like [31] algorithm to describe the fast electrons, while the background electrons are given a hybrid description [32].

The distribution function of the fast electrons is expressed as a truncated spherical harmonic expansion with terms of the form $f_{l}^{m}(x, y, p) P_{l}^{m}(\cos \theta) \exp (\operatorname{im} \phi)$. Substituting this expansion into the VFP equation yields a set of equations for the $f_{l}^{m}(x, y, p)$ coefficients. A brief description of the solution of the VFP equations by means of the KALOS algorithm is given in [31].

This code was previously used in the work reported in $[21,33]$, and has been extended to include the collisional drag on the fast electrons.

The reflective boundaries are used in both $x$ and $y$. For these simulations a box of 4800 cells in $x$ and 40 cells in $y$ where used, and a cell size of $0.2 \mu \mathrm{m}$ was set in both $x$ and $y$. Fast electrons were injected with an isotropic angular distribution from a region in the center of the bottom edge of the simulation box, i.e., the $y$ direction represents the thickness of the foil.

The laser pulse had a FWHM duration of $80 \mathrm{fs}$, an intensity of $5 \times 10^{19} \mathrm{~W} / \mathrm{cm}^{2}$, and a FWHM spot diameter of $6 \mu \mathrm{m}$. The laser to fast electron energy conversion efficiency was set to $20 \%$. The target consisted of a $5.7 \mu \mathrm{m}$ thick $\mathrm{Al}$, with a $2.3 \mu \mathrm{m}$ thick $\mathrm{CH}$ in order to model the rear dielectric coating. In the code, the material properties, including the resistivity curve, the specific heat capacity curve, and the collisional effect on the fast electrons, i.e., scattering and drag, are treated in the same way as previously modeled in $\mathrm{CH}$ and $\mathrm{Al}$ materials [32]. The transverse extent of the coating was taken to cover the entire simulation box. The interface between the metal and the dielectric coating is assumed to be absolutely sharp, in order to model the strong adhesion of the dielectric layer to the metal substrate which characterized the coated targets adopted in our experiment. Simulations are run up to 800 fs.
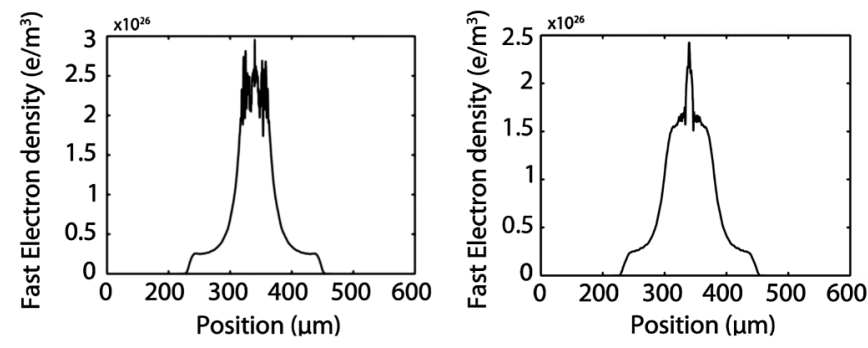

FIG. 2. Comparison of the density profile of the fast electron bunch behind the target and propagating forward as calculated by LEDA in the two cases of uncoated (left) and dielectric rearcoated (right) target. The profile without coating exhibits a top hat profile characterized by strong modulations. In contrast, the profile obtained from the coated target shows a single narrow peak superimposed on a smoother bell-shaped profile. 
The plots of Fig. 2 show the transverse profiles of the fast electron density at the rear surface of the target at $750 \mathrm{fs}$, i.e., a substantial time after the laser pulse has completed its interaction. These plots are representative of the general time history of the simulations and show some important differences between coated and uncoated targets. In particular, in the no-lacquer target the density of fast electrons shows strong fluctuations over a region that is $100 \mu \mathrm{m}$ in diameter. These features are consistent with the measured filament-like structures clearly visible in the image of Fig. 1 (left). In addition, there is a smooth penumbra of fast electron expansion in the transverse direction up to around $200 \mu \mathrm{m}$ in diameter.

In contrast, in the case of the coated target, a central spike is clearly visible on the axis in the plot of Fig. 1 (right). This spike represents a channel of higher density of fast electrons of a few tens of $\mu \mathrm{m}$ in diameter inside a lower density, larger beam that extends up to $100 \mu \mathrm{m}$ in diameter. In this case too, a smooth penumbra of fast electrons extends for approximately $200 \mu \mathrm{m}$ in diameter, similar to the no-lacquer case simulation.

According to these simulations, the rear coating is expected to reduce propagation in the outer region of the lower energy electrons across the interface, allowing only the higher energy fast electrons propagating close to the

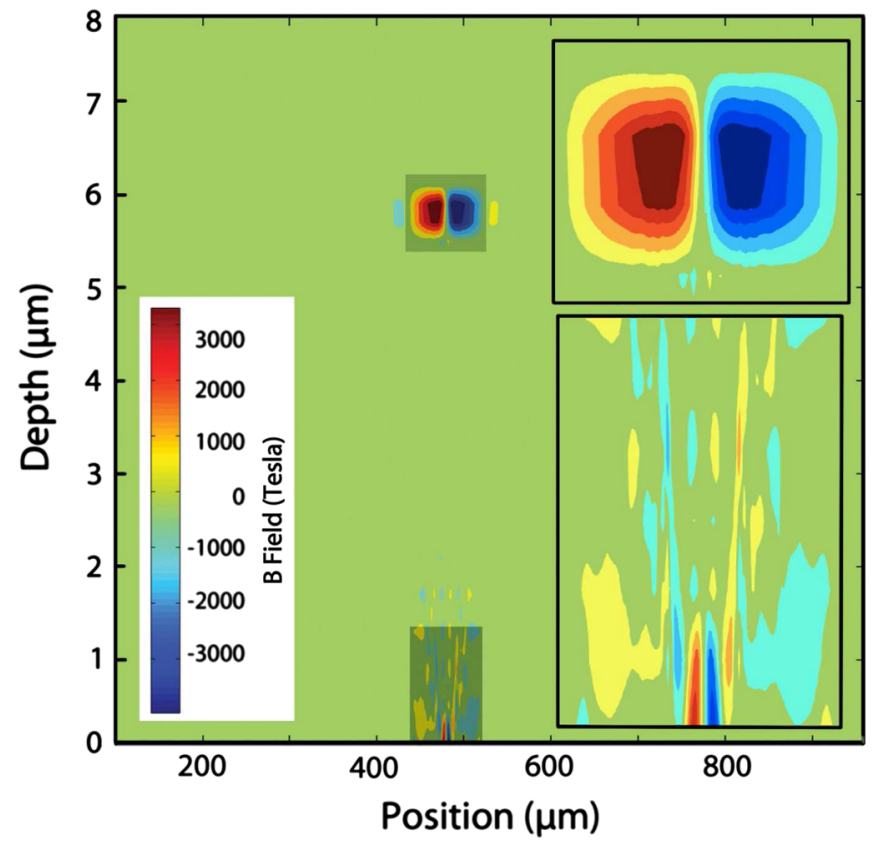

FIG. 3. Map of the magnetic field generated by the interaction of a fast electron beam with a dielectric rear-coated metal target. In the simulation the laser pulse impinges from the bottom of the image on the metal side, where a weak magnetic field structure is also visible (see also bottom-right inset). A much stronger field, greater than 3000 Tesla, is visible at a target depth of $5.7 \mu \mathrm{m}$ (see also top-right inset), where the interface between the metal and the dielectric layer is located. axis to leak efficiently through the target. A detailed analysis of the simulation results suggests that this suppression arises from the onset of a large scale quasistatic B-field which is generated at the interface as described in [20]. The shape and intensity of the B-field is plotted in Fig. 3.

It can be shown that this field originates from the resistivity gradient via the $\mathbf{j}_{\mathbf{f}} \times \nabla \eta$ terms in the induction equation, and this effect has also some similarities to that described in [21]. Because of this strong magnetic field, fast electrons in the peripheral portion of the bunch become magnetized and are confined in the magnetic field region. On the axis the field vanishes and a single intense spike of fast electrons is free to propagate through, dominating the transverse fast electron distribution as shown in Fig. 2 (right). This narrow fast electron bunch will propagate through the dielectric layer up to the target rear side and drive the sheath fields over a much narrower region. This effect is a clear signature of the growth of a magnetic field at the metal-dielectric interface and is fully consistent with the observed proton beam collimation demonstrated by the direct comparison of Fig. 1.

In conclusion, our measurements confirm that dielectric coatings can indeed strongly modify the fast electron transport but, compared with previous results, they also show that the effect of dielectric coating depends strongly on the quality of the metal-dielectric interface. In our experiment, a crucial role was played by the sharp metaldielectric interface achieved with lacquer coating, which enabled us to rule out vacuum gaps as a possible source of fast electron beam inhibition. Our results additionally show that dielectric coatings may be considered as a possible tool to control fast electrons transport to modify the properties of laser-accelerated proton bunches.

\section{ACKNOWLEDGMENTS}

This work was carried out in the framework of the HiPER Project and was partially supported by the MIUR-FIRB project "SPARX", by the MIUR-PRIN2007 project "Studio della generazione di elettroni veloci ...", and by the INFN project PLASMONX. We also acknowledge support from the ESF COST Action MP0601. Access to the IOQ installation was supported by LASERLAB. We wish to acknowledge the JETI laser crew for their invaluable support and the DFG (German Science Foundation). We also acknowledge enlightening discussion with Antonio Giulietti. The present work is part of the "High Field Photonics" CNR Research Unit.

[1] M. Tabak et al., Phys. Plasmas 1, 1626 (1994).

[2] A. Rousse et al., Phys. Rev. E 50, 2200 (1994).

[3] L. A. Gizzi et al., Phys. Rev. Lett. 76, 2278 (1996).

[4] L. A. Gizzi et al., Plasma Phys. Controlled Fusion 49, B211 (2007).

[5] L. Romagnani et al., Phys. Rev. Lett. 95, 195001 (2005). 
[6] F. Cornolti, F. Ceccherini, S. Betti, and F. Pegoraro, Phys. Rev. E 71, 056407 (2005).

[7] S. Betti, F. Ceccherini, F. Cornolti, and F. Pegoraro, Plasma Phys. Controlled Fusion 47, 521 (2005).

[8] J. Fuchs et al., Nature Phys. 2, 48 (2005).

[9] R. A. Snavely et al., Phys. Rev. Lett. 85, 2945 (2000).

[10] M. Roth et al., Phys. Rev. Lett. 86, 436 (2001).

[11] R. A. Snavely et al., Phys. Plasmas 14, 092703 (2007).

[12] M. Borghesi et al., Phys. Rev. Lett. 92, 055003 (2004).

[13] H. Alfven, Phys. Rev. 55, 425 (1939).

[14] A. R. Bell, J. R. Davies, and S. M. Guerin, Plasma Phys. Controlled Fusion 39, 653 (1997).

[15] F. Pisani, Phys. Rev. E 62, R5927 (2000).

[16] D. Batani et al., Phys. Rev. Lett. 94, 055004 (2005).

[17] Y. T. Li et al., Phys. Rev. E 72, 066404 (2005).

[18] J. Fuchs et al., Phys. Rev. Lett. 91, 255002 (2003).

[19] M. Storm et al., Phys. Rev. Lett. 102, 235004 (2009).
[20] A. R. Bell, J. R. Davies, and S. M. Guerin, Phys. Rev. E 58, 2471 (1998).

[21] A.P. L. Robinson and M. Sherlock, Phys. Plasmas 14, 083105 (2007).

[22] S. Kar et al., Phys. Rev. Lett. 102, 055001 (2009).

[23] M. S. Wei et al., Phys. Plasmas 13, 123101 (2006).

[24] S. Betti et al., Phys. Plasmas 16, 100701 (2009).

[25] R. J. Mason, Phys. Rev. Lett. 42, 239 (1979).

[26] E. Breschi et al., Nucl. Instrum. Methods Phys. Res., Sect. A 522, 190 (2004).

[27] S. Agostinelli et al., Nucl. Instrum. Methods Phys. Res., Sect. A 506, 250 (2003).

[28] freely available online at http://www.srim.org.

[29] A. Andreev et al., New J. Phys. 12, 045007 (2010).

[30] X. H. Yuan et al., New J. Phys. 12, 063018 (2010).

[31] A. R. Bell et al., Plasma Phys. Controlled Fusion 48, R37 (2006).

[32] J. R. Davies, Phys. Rev. E 65, 026407 (2002).

[33] A. P. L. Robinson, Phys. Rev. Lett. 100, 025002 (2008). 